\title{
Steuerung der Staatsverschuldung durch Verfassungsrecht im Widerstreit
}

von Werner Heun

Die Ironie des Schicksals will es, dass gerade im Moment krisenbedingter Neuverschuldung in schwindelerregender Höhe die deutsche Verfassungspolitik fiskalische Defizite weitgehend zu unterbinden sucht. Jedoch ist ein allgemeiner Verzicht auf staatliche Neuverschuldung zum einen wirtschaftswissenschaftlich wie politökonomisch nur schwer zu rechtfertigen, zum anderen weist die neue „Schuldenbremse“ im Grundgesetz (wie auch äquivalente Institutionen in den USA und der Schweiz) eine Reihe von Möglichkeiten der Unterwanderung auf, die schon von der neuen Regierungskoalition beinahe zur Finanzierung unnötiger Steuersenkungen heranzogen worden wäre. Anhand einer Zusammenfassung der ökonomischen und verfassungsnormativen Argumente für und wider eine rechtliche Beschränkung der Nettokreditaufnahme sowie im Vergleich mit bestehenden Regelungen in anderen Staaten soll verdeutlicht werden, dass die finanzverfassungsrechtlichen Bestimmungen der „Föderalismusreform II“ auf fragwürdigen Annahmen beruhen und funktional nur schwer haltbar sind.

In an unusual twist of irony, the German attempt to restrict severely the ability of the state to take on new debt has been flanked by soaring public deficits in the context of the ongoing economic and financial crisis. In addition, a general renunciation of new debt is neither economically justifiable nor is the Grundgesetz's new Schuldenbremse (like its counterparts in many US states and Switzerland) as stringent as its proponents claim. By summarising the economic as well as constitutional-normative arguments for and against a legal limitation of the level of new public debt, and by drawing comparisons to existing frameworks in other countries, this article aims to show that the financial-constitutional provisions of the "Föderalismusreform II" are based on questionable assumptions and are, furthermore, hardly tenable from a functional perspective.

\section{Einleitung}

Die aktuelle Finanzkrise hat die Staatsverschuldung weltweit geradezu explodieren lassen. Die Stabilisierung des Banken- und Finanzsystems und die umfangreichen Konjunkturprogramme zur Überwindung des realwirtschaftlichen 
Einbruchs erfordern jeweils schwindelerregende Summen ${ }^{1}$, die allseits als unabdingbar akzeptiert und zugleich als Grundlage von Schreckensgemälden und Horrorszenarien instrumentalisiert werden. Die Ironie des Schicksals will es, dass ausgerechnet auf dem Höhepunkt einer neuen Verschuldenswelle bisher ungekannten Ausmaßes die deutsche Verfassungspolitik sich zu dem Ansinnen verstiegen hat, die Verschuldung verfassungsrechtlich nahezu völlig verbieten zu wollen. Die Diskrepanz zwischen verfassungsrechtlichem Totalverbot und faktischer Verschuldungsausweitung könnte kaum größer sein. Der Wahnsinn hat Methode - die Frage ist nur auf welcher Seite der Verschuldungsmedaille, der normativen oder der realen. In dieser Situation ist es sinnvoll, sich noch einmal ganz grundsätzlich der Rationalität und Problematik der Staatverschuldung wie des Sinns ihrer normativen Regelung in einer Verfassung zu vergewissern.

\section{Staatsfinanzierung durch Staatsverschuldung - polit-ökonomische Determinanten und Restriktionen}

\section{Alternative Finanzierung des Staates durch Steuern und Kredite}

Der Staat kann sich die zur Finanzierung seiner Aufgaben notwendigen Finanzmittel im Wesentlichen entweder durch die Erhebung von öffentlichen Abgaben, d.h. heute vornehmlich von Steuern, oder durch Kreditaufnahme verschaffen. ${ }^{2}$ Während die Steuererhebung als Finanzierungsinstrument selbst kaum in Frage gestellt wird, unterliegt die Staatsverschuldung spätestens seit David Hume ${ }^{3}$ und Adam Smith ${ }^{4}$ scharfer Kritik, die sich durch das 19. Jahrhundert bis heute hindurchzieht ${ }^{5}$. Der hohe Schuldenstand und das ebenfalls extreme staatliche Defizit, das durch die Nettoneuverschuldung definiert ist, verschärfen die öffentliche Kritik in Politik und Wissenschaft, die vor kurzem in die verfassungsrechtliche Verankerung einer Schuldenbremse mündete. Allerdings bleibt diese Kritik viel-

1 Aktuelle Übersicht für die EU European Commission: European Economic Forecast, Autumn 2009, $50 \mathrm{ff}$.

2 Für die Beurteilung sind daher die differentiellen Wirkungen der Verschuldung im Vergleich zur Steuerfinanzierung maßgebend.

3 Hume, D.: Of Public Credit, in: ders.: The Essays Moral, Political and Literary (1742). Part. II, IX; dt. auch in: Diehl, K./Mombert, P. (Hg.): Das Staatsschuldenproblem, Frankfurt/M. u. a., 1980, $30 \mathrm{ff}$.

4 Smith, A.: An Inquiry into the Nature and Causes of the Wealth of Nations, 5. Aufl., London u.a., 1789 (zuerst 1776), bk. V, ch. 3; dt. 1978, 781 ff., hier 798 f.

5 Vgl. den Überblick bei Stern, K.: Das Staatsrecht der Bundesrepublik Deutschland, Bd. II, München, $1980,1264 \mathrm{ff}$. 
fach äußerst pauschal, obwohl eine vielfältige Differenzierung notwendig wäre, die dann ganz andere verfassungsrechtliche Folgerungen nahelegt.

\section{Intertemporale Budgetbeschränkung}

Da die Aufnahme von Krediten die Staatsfinanzierung sichern soll, ist das Interesse des Staates und der politischen Entscheidungsträger darauf gerichtet, dass die Staatsverschuldung langfristig durch den Staatshaushalt finanzierbar bleiben muss, was als intertemporale Budgetbeschränkung bezeichnet wird. Dabei muss schon vorab gerade für Juristen, die sich oftmals von den absoluten Zahlen berauschen lassen, verdeutlicht werden, dass für die Beurteilung der Staatsverschuldung allein die Relation zum Bruttoinlandsprodukt (BIP) maßgebend ist. Die Begrenzung der Staatsverschuldung auf bestimmte Prozentzahlen des BIP durch die Europäische Union ist der juristisch klarste Ausdruck dieser Erkenntnis. Von der Relation zum BIP hängt dementsprechend auch die mittel- und langfristige Finanzierbarkeit ab.

Sofern die Wachstumsrate der Staatsverschuldung derjenigen des Produktionspotentials entspricht, wird eine Erhöhung der Schuldenquote oder eine Einschränkung der Ausgabenspielräume nicht bewirkt. Im Fall dieser sog. potentialorientierten Verschuldung ist zwar die steigende Verschuldung mit höheren Zinsausgaben verbunden, deren Anteil am gesamten Haushaltsvolumen strebt aber einem Grenzwert zu. ${ }^{6}$ In jüngster Zeit ist das Konzept der fiscal sustainability in den Vordergrund getreten, wonach die Zinszahlungen bei gleichbleibendem BIP aus dem Überschuss der sonstigen Staatseinnahmen über die Staatsausgaben finanziert werden müssen, wenn der absolute Schuldenstand nicht ansteigen soll. $^{7}$ Im Fall einer stationären Wirtschaft ist daher im Grunde jede Staatsverschuldung auf Dauer nicht finanzierbar, wenn die Staatsausgaben unter Einschluss der Zinsen nicht voll durch die nichtkreditären Einnahmen gedeckt sind. Das Bild ändert sich freilich grundlegend in einer dynamischen, durch langfristiges Wachstum geprägten Wirtschaft. In diesem Fall kommt es nämlich nicht mehr auf das absolute Schuldenniveau an, sondern lediglich auf die Stabilisierung der Schuldenquote, sofern diese im Anfangszeitpunkt tragbar ist. Die

6 Klassisch Domar, E. D.: The Burden of the Debt and National Income, in: American Economic Review, 34/4 (1944), 798-827; s. a. Scherf, $W$. Öffentliche Finanzen, Stuttgart, 2009, 432 ff.

7 Grundlegend Blanchard, O. u. a.: The Sustainability of Fiscal Policy, in: OECD-Economic Studies, 15 (1990), 7-36; s. a. Wiss. Beirat beim BMF: Nachhaltigkeit in der Finanzpolitik, Berlin, 2001; Afonso, A.: Fiscal Sustainability: The Unpleasant European Case, in: Finanzarchiv, 61/1 (2005), 19-44. 
Schuldenquote wächst danach nicht weiter an, wenn der Realzinssatz die reale Wachstumsrate nicht übersteigt. In diesem Fall ist der Grundsatz der Nachhaltigkeit (sustainability) nicht verletzt und der Staat gerät auch langfristig nicht in die Schuldenklemme.

Es kann deshalb nicht oft genug betont werden, dass die Staatsverschuldung und ihre Gefahren eine Frage der Proportionalität des Defizits und des Schuldenstands zum BIP ist. Die aktuellen Staatsdefizite sind auf Dauer nicht tragbar, da sie markant über den mittelfristigen Wachstumsraten liegen, in den USA sogar bei fast $10 \%$ des BIP $2009^{8}$, in Deutschland allerdings (nur) 3,5\% 2009 und voraussichtlich $5 \% 2010^{9}$. Sieht man von den USA ab, sind diese Verhältnisse aber immer noch nicht allzu besorgniserregend, wenn die Defizite zügig wieder auf ein der Wachstumsrate entsprechendes Niveau zurückgeführt werden. Auch ist es vor Ausbruch der Krise einer ganzen Reihe von Staaten gelungen, innerhalb vergleichsweise kurzer Zeit die Schuldenquote in bemerkenswertem Umfang zu reduzieren ${ }^{10}$ und das Defizit sogar - besonders spektakulär in den USA - zeitweise auf null ${ }^{11}$ zu senken.

Die Sicherung der Nachhaltigkeit ist zweifellos ein sinnvolles Ziel der Verfassungspolitik. Die Abhängigkeit der Nachhaltigkeit von fluktuierendem Realzins wie realer schwankender Wachstumsrate erschwert allerdings oder verhindert sogar eine praktikable Umsetzung in verbindliche Verfassungsnormen. Die verfassungsrechtliche Regelung der neuen Schuldenbremse mit dem Ziel der Reduktion auf nahezu Null schießt indes weit über die Sicherung der Nachhaltigkeit hinaus. Die Begrenzung durch die Regeln des Art. 126 AEUV (= 104 EGV a.F.) und des Stabilitätspaktes, die das Defizit auf $3 \%$ des nominalen BIP und den Schuldenstand auf $60 \%$ des BIP beschränken ${ }^{12}$, kommen bei aller Willkürlich-

8 Congressional Budget Office (CBO): Monthly Budget Review Fiscal Year 2009, 07. 10. 2009; Anfang des Jahres lag die Schätzung sogar noch bei $13,1 \%$, s. CBO: Preliminary Analysis of the President's Budget, March 2009, 2.

9 European Commission, a.a. O., 85.

10 Vgl. Wagschal, U.: Erfolgreiche Budgetkonsolidierungen im internationalen Vergleich, Gütersloh, 2006.

11 Unter Clinton ist das Defizit des Bundes in einen Rekordüberschuss von 4,1 \% des BIP im Jahr 2000 verwandelt worden, s. a. Wagschal, $U$, a. a. O., $103 \mathrm{ff}$.

12 Verordnung (EG) Nr. 1466/97 des Rates vom 07.07. 1997 über den Ausbau der haushaltspolitischen Überwachung und der Überwachung und Koordinierung der Wirtschaftspolitiken, ABlEU Nr. L 209/1 v. 02.08. 1997; Verordnung (EG) Nr. 1467/97 des Rates vom 07.07. 1997 über die Beschleunigung und Klärung des Verfahrens bei einem übermäßigen Defizit, ABlEU Nr. L 209/6 v. 02.08. 1997; dazu Sutter, M.: Der Stabilitäts- und Wachstumspakt in der Europäischen Währungsunion, Baden-Baden, 2000; Hüpertz, M./Verdun, A.: The Dog that Would Never Bite?, MPIfG Working Paper 03/12, 2003, 3 ff.; 
keit der gegriffenen Zahlen, die zudem nicht wirklich miteinander kompatibel $\operatorname{sind}^{13}$, angemessenen Werten, die typisierend eine Nachhaltigkeitsgarantie bewirken können, sehr viel eher nahe. Als zwar sehr grober Maßstab können diese Regeln jedenfalls unter dem Gesichtspunkt der Sicherung der Tragbarkeit eine gewisse Rationalität beanspruchen - im klaren Gegensatz zur Schuldenbremse.

\section{Zukunftsbelastung durch Staatsverschuldung}

Anders als Steuern und andere Abgaben, die den Bürger gegenwärtig belasten und eine endgültige Deckung der Ausgaben bewirken, gilt die staatliche Kreditaufnahme spätestens seit Adam Smith ${ }^{14}$ als eine Zukunftsbelastung, die rechtfertigungsbedürftig ist. Die Zukunftsbelastung darf allerdings nicht mit einer Lastenverschiebung auf die künftigen Steuerzahler in Höhe der Zins- und Tilgungslast gleichgesetzt werden, obwohl diese Auffassung offenbar nicht nur bei Juristen $^{15}$, sondern auch bei Ökonomen ${ }^{16}$ und der Öffentlichen Meinung sowie der Politik nicht auszurotten ist. Die mit pejorativen Hintersinn so genannte Neue Orthodoxie ${ }^{17}$ hat vielmehr zu Recht darauf hingewiesen, dass eine zeitliche Lastenverschiebung jedenfalls in geschlossenen Volkswirtschaften in dieser Form nicht eintreten kann, da Tilgungs- und Zinsleistungen jeweils von den Mitgliedern, den Steuerzahlern, derselben Gesellschaft aufgebracht werden, deren Mitglieder auch zugleich die Empfänger der Zahlungen als Kreditgläubiger sind. "We owe it to ourselves " ist die plastische Formulierung dieses Tatbestandes. ${ }^{18}$ Die volkswirtschaftlichen Ressourcen können zu einem gegebenen Zeitpunkt jeweils immer nur einmal in Anspruch genommen werden. Unter gesamtwirt-

Beemtsma, R.M.W./Debrun, X.: Implementing the Stability and Growth Pact. Enforcement and Procedural Flexibility, ECB Working Paper 433, Jan. 2005.

13 Vgl. Scherf, $W$., a. a. O., 433.

14 Smith, $A$, a. a. O.

15 Vgl. etwa Gröpl, C.: Haushaltsrecht und Reform, Tübingen, 2001, 442, 444; Kirchhof, P.: Verfassungsrechtliche Grenzen der Steuerlast und der Staatsverschuldung, in: Frankfurter Institut - Stiftung Marktwirtschaft und Politik (Hg.): Schranken gegen Staatsverschuldung und Steuerlast, Bad Homburg, 1996, 51-77, hier $74 \mathrm{f}$.

16 S. etwa Sinn, H.-W.: Kasino-Kapitalismus, Berlin, 2009, 11.

17 Buchanan, J.M.: Public Principles of Public Debt, Homewood, 1958, 4 ff.

18 Lerner, A.P.: The Burden of the National Debt, in: Income, Employment and Public Policy, New York, 1948, 255-275, hier 256; gekürzt in: Ferguson, J. M. (Hg.): Public Debt and Future Generations, Richmond, 1964, 16 ff; auch diese Auffassung findet freilich schon im 18. Jh. Vorläufer, Buchanan J.M., a. a. O., $17 \mathrm{ff}$. 
schaftlicher Perspektive ist das im Grunde auch unbestritten. ${ }^{19}$ Man hat diese gesamtwirtschaftliche Betrachtung mit dem geradezu abwegigen Argument zu kritisieren versucht, dieser Sicht liege ein „totalitäres Staatsverständnis“ zugrunde. ${ }^{20}$ Offenbar haben diese Autoren keinen Begriff vom Totalitarismus ${ }^{21}$, vielmehr muss man wohl eine Verwechslung mit Kollektivismus oder ähnlichem unterstellen. ${ }^{22}$ Auch das ist freilich nur als Denunziation zu verstehen, denn man müsste dann im Grunde jede gesamtwirtschaftliche Perspektive als kollektivistisch verdammen.

Als Antithese zur Neuen Orthodoxie ist der Ansatz zur Begründung einer Zukunftsbelastung durch den individualistischen sog. Utility Approach James Buchanans konzipiert. ${ }^{23}$ Danach soll eine Zukunftsbelastung dadurch eintreten, dass der Einzelne eine individuelle Nutzeneinbuße durch die zukünftige Zwangsbelastung durch Steuern erleide, wohingegen der Kreditgeber in der Gegenwart sein Kapital freiwillig und im Austausch gegen einen entsprechenden Rückzahlungsanspruch zur Verfügung stellt. Die Zukunftsbelastung besteht also ausschließlich in dem Zwangscharakter der späteren Steuerfinanzierung und den damit verbundenen Nutzeneinbußen. Abgesehen davon, dass auch diese Effekte einen Zustand der Vollbeschäftigung voraussetzen ${ }^{24}$ und auf der Annahme der sog. Ricardo'schen Schuldillusion beruhen ${ }^{25}$, ist dieser Ansatz dem Einwand ausgesetzt, dass sich Nutzengewinn und -einbuße weder intersubjektiv vergleichen noch in sonstiger Weise messen lassen und damit inoperationabel sind. Die Lasteffekte beziehen sich auf ,gefühlte, aber nicht auf reale Belastungen der Wirtschaftssub-

19 Vgl. Buchanan J.M., a. a. O., 35; Tobin, J.: The Burden of the Public Debt: A Review Article, in: Journal of Finance, 20/4 (1965), 679-683, hier $679 \mathrm{f}$.

20 Vanberg, V./Buchanan, J.M.: Organization Theory and Fiscal Economics: Society, State and Public Debt, in: Journal of Law, Economics and Organization, 2/2 (1986), 215-227, hier 220; dem folgend Blankart, C. B.: Öffentliche Finanzen in der Demokratie, 7. Aufl., München, 2008, $358 \mathrm{f}$.

21 Vgl. hier nur zur Übersicht m.w. N.: Jesse, E.: Totalitarismus, in: Heun, W. u. a. (eds.): Evangelisches Staatslexikon, Stuttgart, 2006, Sp. $2474 \mathrm{ff}$.

22 Vanberg, V./Buchanan, J.M., a. a.O., $216 \mathrm{f}$. sprechen auch zunächst von organizistischen Vorstellungen; siehe auch schon Buchanan, J.M., a. a. O., $35 \mathrm{f}$.

23 Buchanan, J.M., a. a. O., $31 \mathrm{ff}$.

24 Ebd., $123 \mathrm{ff}$.

25 Vgl. Gandenberger, O.: Intertemporale Wirkungen der Staatsverschuldung, in: Haller, H./Albers, W. (Hg.): Probleme der Staatsverschuldung, Berlin, 1972, 189-214, hier 208 ff. 
jekte“26. Dieses Konzept eignet sich deswegen kaum als Grundlage gesamtstaatlicher verfassungsrechtlicher Regelungen. ${ }^{27}$

Am weitesten verbreitet ist daher der makroökonomisch orientierte Aggregate Investment Approach, der auf differentielle Wachstumseffekte abstellt. ${ }^{28}$ Ebenfalls Vollbeschäftigung vorausgesetzt, kann der Staat seine Ausgaben nur zu Lasten entweder des privaten Konsums oder der privaten Investitionen finanzieren. Während die Steuerfinanzierung - jedenfalls bei linearer Aufhebung des Einkommensteuertarifs - den privaten Konsum belastet, wird durch die staatliche Kreditaufnahme privates Anlagekapital in Anspruch genommen, so dass über die Anhebung des Zinsniveaus private Investitionen verdrängt werden (crowding$o u t)$. Die Zukunftsbelastung liegt darin, dass infolge der Verdrängung ein geringeres Produktionspotential gebildet und der Zukunft ein geringer Kapitalstock vererbt wird, was den Konsum verringert. Die im Modell unterstellte vollständige Verdrängung ist freilich selbst im Fall der Vollbeschäftigung außerordentlich zweifelhaft, da das Zinsniveau durch eine Vielzahl von der Staatsverschuldung unabhängiger Variabler bestimmt wird und auch die durch die Kredite finanzierten Ausgaben, die dem Kapitalmarkt wieder Mittel zuführen, berücksichtigt werden muss. ${ }^{29}$ Unter der Annahme uneingeschränkt rationaler Erwartungen sind Steuern und Staatsverschuldung darüber hinaus völlig äquivalent, da die Steuerzahler die künftigen Steuern antizipieren (Ricardo-Barro-Äquivalenztheorem). ${ }^{30}$ Sowohl die diesem Theorem zugrunde gelegte Annahme rationaler Erwartungen als auch die notwendige Voraussetzung eines unendlichen generationsübergreifenden Zeithorizonts, der etwa bei älteren Wählern, die in absehbarer Zeit nicht mehr (voll) der Steuer unterliegen, gerade nicht unterstellt werden kann, sind

26 Scherf, $W$., a. a. O., 426.

27 Übersehen bei Wendt, R./Elicker, M.: Staatsverschuldung und intertemporäre Lastengerechtigkeit, in: Deutsches Verwaltungsblatt, 116/7 (2001), 497-504, hier 498.

28 Grundlegend Modigliani, F.: Long-run Implications of Alternative Fiscal Policies and the Burden of the National Debt, in: The Economic Journal, 71/284 (1961), 730-755; Vickrey, W.: The Burden of the Public Debt: Comment, in: American Economic Review, 51/1 (1961), 132-137; Friedmann, B. J.: Crowding Out or Crowding in? Economic Consequences of Financing Government Refints, in: Brookings Papers on Economic Activity, 3 (1978), 593-654.

29 Vgl. Duwendag, D.: Staatsverschuldung - Notwendigkeit und Gefahren, Baden-Baden, 1983, $82 \mathrm{ff}$., plastisch ist auch von Fontänen-Theorie die Rede; vgl. auch Heun, W.: Staatsverschuldung und Grundgesetz, in: Die Verwaltung, 18/1 (1985), 1-28, hier 11 f. m.w. N.

30 Ricardo, D.: On the Principles of Political Economy and Taxation, 1817, dt. 1972, 123 ff., 182 ff.; Barro, R. J.: Are Government Bonds Net Wealth?, in: Journal of Political Economy, 82/6 (1974), 1095-1117; ders.: The Ricardian Approach to Budget Deficits, in: Journal of Economic Perspectives, $3 / 2$ (1989), $37-54$. 
freilich zweifelhaft, so dass eine realistische Theorie der Staatsverschuldung darauf nicht aufbauen sollte. Zudem sind wiederum die Investitionen und die auf diese gerichtete Kreditnachfrage von zahlreichen anderen Faktoren abhängig. Es muss daher nicht verwundern, dass sich die theoretischen Annahmen empirisch praktisch nicht nachweisen lassen. Die empirischen Untersuchungen lassen vielmehr keinen klaren Schluss zu. ${ }^{31}$ Das gilt schon für die erste Stufe der Beeinflussung des Zinsniveaus. Die zweite Stufe, nämlich die Verdrängung der Investitionen entzieht sich einer empirischen Nachprüfung noch stärker. Trotz dieser erheblichen Einschränkungen bleibt dieser Ansatz immer noch die plausibelste Hypothese, die im Rahmen einer gesamtwirtschaftlichen Perspektive immerhin eine Tendenzaussage erlaubt. Schließlich ist hier eine stringente Verknüpfung mit den staatlichen Investitionsausgaben als Kompensation der Verdrängung privater Investitionen möglich, die auch verfassungsrechtlich operationabel ist.

\section{Interpersonelle, funktionelle und institutionelle Verteilungswirkungen}

Gegenüber den intertemporalen Verteilungswirkungen treten die interpersonellen Verteilungswirkungen in der Diskussion ganz zurück. Auch hier gilt, dass prima facie Eindrücke in die Irre führen. Die These einer unsozialen Verteilung beruht danach auf dem Umstand, dass die Kreditgläubiger und damit die Zinsempfänger den höheren Einkommensschichten angehören, die Steuern zur Finanzierung der Zinszahlungen aber von allen Steuerzahlern aufgebracht werden. ${ }^{32}$ Dieses Argument ist schon deswegen verfehlt, weil die Gläubiger ihr Geldkapital ebenso anderen privaten Wirtschaftssubjekten gegen Zinszahlungen zur Verfügung stellen können, die Staatsverschuldung also nicht die Ursache ihres Zinsanspruchs ist. Allein die Erhöhung des Zinseinkommens durch höhere Zinssätze infolge

31 So das resignative Ergebnis von Elmendorf, D.W./Maukiw, G.: Government Debt, in: Taylor, J. B./Woodford, M (Hg.): Handbook of Macroeconomics, Bd. 1 C, Amsterdam, 1999, 1615-1669, hier 1656 f.; vgl. außerdem: Plosser, C. I.: Government Financing Decisions and Asset Returns, in: Journal of Monetary Economics, 9/3 (1982), 325-352; Evans, P.: Do Budget Deficits Raise Nominal Interest Rates?, in: Journal of Monetary Economics, 20/2 (1987), 281-300; Ball, L./Mankiw, G.: What Do Budget Deficits Do?, NBER Working Paper 5263, 1995, 7; Engen, E. M./Hubbard, R. G.: Federal Government Debt and Interest Rates, NBER Working Paper 10681, 2004; Gale, W. G./Orszag, P. R.: Budget Deficits, Notional Saving and Interest Rates, 2004; Kinoshita, N.: Government Debt and Interest Rates, IMF Working Paper 2006/63.

32 Vgl. m.w. N. auch zum Folgenden Andel, N.: Zur These von den unsozialen Verteilungswirkungen öffentlicher Schulden, in: Public Finance, 24/1 (1969), 69-79; Gandenberger, G.: Öffentlicher Kredit und Einkommensverteilung, in: Finanzarchiv, 29/1 (1970), 1-16; Kurz, R./Rall, L.: Interpersonelle und intertemporale Verteilungswirkungen öffentlicher Verschuldung, Tübingen, 1983, $5 \mathrm{ff}$., $25 \mathrm{ff}$. mit eingehenden empirischen Untersuchungen. 
staatlicher Kreditaufnahme unter der Annahme der Vollbeschäftigung kann einen zusätzlichen Einkommenseffekt bewirken. Hier gelten allerdings dieselben auch empirischen - Zweifel an diesem Zinseffekt wie beim crowding out. Außerdem lässt selbst diese Annahme die Verteilung der Steuerlast unberücksichtigt, da die höheren Einkommensschichten und Kreditgläubiger auch in erhöhtem $\mathrm{Ma} ß$ das Steueraufkommen tragen. Die Besteuerung der Zinseinkünfte und die Abschwächungseffekte der Auslandsverschuldung treten hinzu. Soweit sich nach den allgemeinen Verteilungsgleichungen Verschiebungen zugunsten der Unternehmer im Vergleich zu den Arbeitnehmern ergeben, ${ }^{33}$ sind diese Wirkungen gegenläufig zu der damit in Konkurrenz tretenden Verdrängung der Investitionen, unterschätzen die Kreditschöpfungsmöglichkeiten der Finanzmärkte und lassen schließlich die Ausgabenseite der Staatsfinanzierung durch Kreditaufnahmen außer Betracht. Eine auch nur ansatzweise quantifizierbare, klare Beurteilung der Effekte ist daher auch heute kaum möglich. Die Wirkungen begründen deshalb letztlich keine Ablehnung der staatlichen Kreditaufnahme.

\section{Polit-ökonomische Ursachen der Staatsverschuldung}

In der Sicht des Public-Choice-Ansatzes orientieren sich die politischen Entscheidungsträger vornehmlich an ihrem persönlichen Nutzen. ${ }^{34}$ In der demokratischen Ordnung lassen sich insoweit politische (Stimmen-)Gewinne bei zusätzlichen Leistungen erwarten, denen aber Verluste infolge der notwendigen Finanzierung durch belastende Steuern gegenüberstehen. Staatsverschuldung erscheint in dieser Perspektive als Möglichkeit der Verringerung der politischen Kosten der Finanzierungslasten durch eine Verschiebung der Steuern in die Zukunft. ${ }^{35}$ Im Falle rationaler Antizipation dieser künftigen Steuerlast (so das oben angesprochene Ricardo-Barro-Äquivalenztheorem) entfiele dieser Effekt ebenso wie die Zukunftsbelastung. Je stärker die Bürger aufgrund unvollkommener Information einer fiscal illusion unterliegen, umso eher wirkt dieser Effekt. ${ }^{36}$ Informationskosten, begrenzter Zeithorizont vor allem älterer Wähler ${ }^{37}$, der Unterschied zwischen gesamtwirtschaftlicher Steuerbelastung und individuell zu erwartender

33 Darauf stellt ab Scherf, $W$., a. a. O., $419 \mathrm{ff}$.

34 Vgl. hier nur das Standardlehrbuch Müller, D.: Public Choice III, Cambridge, 2003.

35 Grundlegend für diese Perspektive Buchanan, J.M./Wagner, R.: Democracy in Deficit - The Political Legacy of Lord Keynes, New York u. a., 1977, $93 \mathrm{ff}$.

36 Vgl. ebd., $129 \mathrm{ff}$.

37 Vgl. Blankart, C.B., a. a. O., $340 \mathrm{f}$. 
tatsächlicher Belastung lassen jedenfalls darauf schließen, dass staatliche Kreditaufnahme in einem gewissen Maß - dessen Umfang freilich empirisch völlig unklar ist - als insgesamt geringere Belastung denn durch eine Steuer empfunden wird, so dass tendenziell ein polit-ökonomischer Anreiz zur Kreditaufnahme plausibel ist. An dieser Stelle wird noch von denkbaren positiven Wirkungen der Staatsverschuldung aus der Sicht des rationalen Wählers ganz abgesehen.

Institutionelle Strukturen können außerdem die staatliche Verschuldung zusätzlich fördern oder bremsen. Angesichts der Komplexität politischer Entscheidungen über das staatliche Budget sind freilich gesicherte Aussagen außerordentlich schwierig zu treffen. ${ }^{38}$ Gerade die ökonomische Theorie und Modellbildung hat dies durch übergroße Simplizität zu überspielen gesucht. Der Kern mathematisch unterlegter Behauptungen reduziert sich oft auf kaum mehr als Binsenwahrheiten, deren realer Gehalt sich schwer verifizieren lässt. Weder die Vorstellung der politischen Konjunkturzyklen ${ }^{39}$ noch die These, dass linke Parteien eine expansive Fiskalpolitik betreiben ${ }^{40}$, gehen über prima-facie-Plausibilität hinaus ${ }^{41}$; der empirische Nachweis fehlt. Auch die Modelle eines sog. Stellvertreterkriegs nach politischen Wahlen, bei dem keine Interessengruppe zu einer vorzeitigen Reduzierung der Staatsverschuldung bereit ist ${ }^{42}$ und die Vorstellung einer Erhöhung der Staatsschuld, um nachfolgende Regierungen strategisch zu behindern, ${ }^{43}$ können nicht viel Überzeugungskraft für sich mobilisieren.

38 Guter Überblick über die verschiedenen Ansätze Mikosch, H.F./Übelmesser, S.: Staatsverschuldungsunterschiede im internationalen Vergleich und Schlussfolgerungen für Deutschland, in: Perspektiven der Wirtschaftspolitik, 8/4 (2007), 303-334, hier 312 ff.; vgl. auch Woo, J.: Economic Political and Institutional Determinants of Public Deficits, in: Journal of Public Economics, 87/3-4 (2003), 387-426.

39 Nordhaus, W.D.: The Political Business Cycle, in: Review of Economic Studies, 42/2 (1975), 169-190; MacRae, C.D.: A Political Model of the Business Cycle, in: Journal of Political Economy, 85/2 (1977), 239-263; Mink, M./de Haan, J.: Are there Political Budget Cycles in the Euro Area?, in : European Union Politics, 7/2 (2006), 191-211; dagegen Schneider, C.J.: Politischer Opportunismus und Haushaltsdefizite in den westdeutschen Bundesländern, in: Politische Vierteljahresschrift, 48/2 (2007), 221-242.

40 Vgl. Hibbs, D. A.: Political Parties and Macroeconomic Policy, in: American Political Science Review, 71/4 (1977), 1467-1487; ders.: Comment on Beck, in: American Political Science Review, 77/2 (1983), 447-451; ders.: The American Political Economy, Cambridge, MA, 1987, 255 ff.; die Finanzpolitik unter Reagan und Clinton mag jeweils als markantes Gegenbeispiel dienen.

41 Kritisch etwa Wagschal, U.: Staatsverschuldung, Opladen, 1996, 95 ff., 218 ff.; ders. u. a.: Determinanten der Staatsverschuldung in den deutschen Bundesländern, in: Widi 2009, 204-212, hier 208, 206.

42 Alesina, A./Drazen, A.: Why are Stabilizations Delayed?, in: American Economic Review, 81/5 (1991), 1170-1188.

43 Alesina, A./Tabellini, G.: A Positive Theory of Fiscal Deficits and Government Debt, in: Review of Economic Studies, 57/3 (1990), 403-414; bei den republikanischen Administrationen von Reagan und Bush jun. war nicht die Behinderung der Nachfolger das Motiv, sondern generell der durch die Staatsverschuldung erzeugte politische Druck zu Ausgabenkürzungen; vgl. a. Wagschal, U.: Staatsverschuldung, a.a.O., $119 \mathrm{ff}$. 
Plausibler ist dagegen der empirische Vergleich unterschiedlicher institutioneller Strukturen im Hinblick auf die Entwicklung der Staatsverschuldung, die durch qualitative Gründe gestützt werden kann, obwohl auch in diesen Fällen die Untersuchungen kaum mehr als vorsichtige Anhaltspunkte und Tendenzen erkennen lassen. Danach wirken sich eine starke Stellung des Regierungschefs und des jeweiligen Finanzministers im Sinne einer Dämpfung der Staatsverschuldung aus. $^{44}$ Die Wahlsysteme und die davon geprägten Parteiensysteme üben einen noch stärkeren Einfluss aus. Wahlsysteme mit überwiegender Mehrheitswahl oder mehrheitsfördernde Verhältniswahlsysteme steigern die Staatsverschuldung tendenziell weniger als stark proportionale Verhältniswahlsysteme mit einem zersplitterten Parteiensystem, in denen Regierungskoalitionen aus mehreren Parteien bestehen, deren jeweilige Wählerschaft und Klientel nach Wahlen bedient werden müssen. ${ }^{45}$ Die Verschuldungsquoten der OECD-Länder ${ }^{46}$ zeigen freilich, dass diese institutionellen Strukturen allenfalls begrenzte Einflussfaktoren und keine wirklich entscheidenden Determinanten, die viel eher sozioökonomischer Natur sind, darstellen. Die Struktur des politischen Systems der Bundesrepublik ist danach im Übrigen eher verschuldungsavers, ohne dass die Staatsverschuldung dadurch wirklich begrenzt worden wäre. Allerdings bewegen sich die Quoten der Bundesrepublik bisher im Mittelfeld. Eine Steuerung der Staatsverschuldung über verfassungsrechtliche Änderungen der institutionellen Struktur ist weder nötig noch sonderlich erfolgversprechend, zumal sie kaum sinnvoll isoliert im Hinblick auf ein einzelnes Problemfeld vorgenommen werden könnten.

\section{Materielle Begrenzungen durch Verfassungsrecht}

\section{Historische Entwicklung}

Die geringe Determinationskraft institutioneller Strukturen und die polit-ökonomischen Anreize zur Verschuldung haben immer wieder - und gerade in einem

44 Hagen, J.v.: Budgeting Procedures and Fiscal Performance in the European Communities, European Commission Economic Papers 96, 1992; Seils, E.: Haushaltspolitik: Akteure und Institutionen des parlamentarischen Systems der Bundesrepublik im internationalen Vergleich, in: Zeitschrift für Parlamentsfragen, 36/4 (2005), 773-790, hier $780 \mathrm{ff}$.

45 Vgl. v.a. Persson T./Tabellini, G.: The Economic Effects of Constitutions, Cambridge, MA u.a., 2003, insbes. 81 ff., 179 ff. m. w. N.; einschränkend Wagschal, U.: Staatsverschuldung, a. a. O., $126 \mathrm{ff} ., 197 \mathrm{ff}$.

46 OECD: Economic Outlook No. 85, June 2009, Tabelle 27 (Defizitquoten), Tabelle 32 (BruttoSchuldenstandsquoten). 
zur Juridifizierung neigenden System wie der Bundesrepublik - den Versuch einer verfassungsrechtlichen materiellen Begrenzung der Staatsverschuldung herausgefordert.

In Deutschland fanden sich bereits im 19. Jahrhundert materielle Beschränkungen in einzelnen Verfassungen. So wurde ein dringendes oder außerordentliches Staatsbedürfnis als rechtfertigende Ursache verlangt. ${ }^{47}$ Dieses Erfordernis wurde von Art. 73 Reichsverf. 1871 übernommen und in Art. 87 WRV dahingehend verschärft, dass neben dem außerordentlichen Bedarf auch noch aufgenommen wurde, dass die Finanzmittel nur zu werbenden Zwecken verwendet werden durften. ${ }^{48}$ Das Grundgesetz schloss sich dieser Regel mit der Modifizierung an, dass die werbenden Zwecke gestrichen wurden. ${ }^{49}$ Erst 1969 wurde Art. 115 GG grundlegend umgestaltet und hatte in dieser Form vierzig Jahre Bestand.

\section{Die überkommene Regelung des Art. 115 Abs. 1 GG}

Die Kreditbegrenzungsregel des Art. 115 Abs. 1 GG in ihrer 2009 aufgehobenen Fassung enthielt zwei verschiedene, als Grundsatz und Ausnahme konzipierte Regeln für die staatliche Kreditaufnahme.

Grundsätzlich war die Kreditaufnahme in der wirtschaftlichen Normallage auf die Summe der Investitionsausgaben beschränkt. Diese Vorgabe wird vielfach von ökonomischer Seite auch als „Goldene Regel“ bezeichnet. ${ }^{50}$ Ihre ökonomische Logik beruht letztlich auf den Überlegungen des aggregate investment approach zur zukunftsbelastenden Wirkung der Staatverschuldung. ${ }^{51}$ Durch die staatliche Kreditaufnahme verdrängte Privatinvestitionen sollten durch öffentliche Investitionen kompensiert werden. Daneben ließ sich für diese Verwendungsvorgaben auch noch ergänzend das pay-as-you-use-Argument heranziehen $^{52}$, dessen Überzeugungskraft und Reichweite allerdings auch wegen der unterschiedlichen Haltbarkeit und Dauerhaftigkeit öffentlicher Investitionen

47 Als Beispiel: Tit. VII, $\S 12$ Bayer Verf. 1818.

48 Zur Entwicklung dieser Deckungsregel eingehend Höfling, W.: Staatsschuldenrecht, Heidelberg, 1993, $107 \mathrm{ff}$.

49 Vgl. Höfling, W./Rixen, S., in: Bonner Kommentar zum Grundgesetz, Art. 115 (2003), Rn. 1 ff.

50 Sachverständigenrat: Staatsverschuldung wirksam begrenzen, 2007, 50; Pünder, H.: Staatsverschuldung, in: Isensee, J./Kirchhof, P. (Hg.): Handbuch des Staatsrechts für die Bundesrepublik Deutschland, Bd. V, 3. Aufl., Heidelberg, 2007, § 123, hier Rn. 3 f.

51 Heun, W.: Staatsverschuldung, a.a. O., 15; Höfling, $W$.: Staatsschuldenrecht, a. a. O., 189 ff.

52 Vgl. Musgrave, R.A.: Finanztheorie, 2. Aufl. Tübingen, 1969, 523 ff.; Heun, W.: Staatsverschuldung, a. a. O., 18 f. m.w.N. 
begrenzt ist. Gerade aus gesamtwirtschaftlicher Sicht ist dies trotz aller Unschärfen des crowding out eine angemessene Regel. Zwei Fragen wirft das Konzept dieser Regel auf. Zum einen wird teilweise die Äquivalenz der Produktivität öffentlicher und privater Investitionen bezweifelt. Das ist zumindest in dieser Pauschalität nicht haltbar. Empirische Untersuchungen sind zu keinen eindeutigen Ergebnissen gelangt, ${ }^{53}$ was nicht zuletzt an der Schwierigkeit der Messbarkeit des wirtschaftlichen Nutzens öffentlicher und meritorischer Güter liegt. ${ }^{54}$ Aufs Ganze gesehen wird man keinen signifikanten Produktivitätsunterschied feststellen können, der der Kompensationsidee prinzipiell den Boden entzieht. Zum anderen ist die Gleichsetzung des Investitionsbegriffs des Art. 115 Abs. 1 GG mit Bruttoinvestitionen gerügt und als Verschärfung der Ersetzung durch Nettoinvestitionen gefordert worden. ${ }^{55}$ Mit ökonomischer Logik lässt sich dies indes nicht begründen. Die Verdrängungseffekte durch staatliche Kreditaufnahmen betreffen pauschal alle, d.h. generell Bruttoinvestitionen. Die Kompensation durch Nettoinvestitionen schießt daher über das Ziel der Kompensation hinaus. ${ }^{56}$ Die Forderung war daher offensichtlich allein durch das Anliegen motiviert, die Staatsverschuldung um jeden Preis zu reduzieren.

Die „Goldene Regel“" als solche ist freilich bis heute weithin akzeptiert. Weitaus schärferer Kritik unterlag die Ausnahmeregelung des Art. 115 Abs. 1 GG, die im Fall einer Störung des gesamtwirtschaftlichen Gleichgewichts eine Überschreitung der „Goldenen Regel“ erlaubte. Ungeachtet denkbarer Kritik im Detail ist aber zunächst einmal die prinzipielle ökonomische Rechtfertigung dieser Durchbrechung festzuhalten. Sie ist nämlich sogar doppelt fundiert. Erstens geht die Verdrängungsthese - wie im Übrigen auch der Nutzenansatz - von der Annahme einer Vollauslastung des Produktionspotentials, insbesondere Vollbeschäftigung aus. Diese Voraussetzung entfällt jedoch in einer Rezession und damit eben auch

53 Vgl. aus der Literatur z.B. Ford, R./Poret, P.: Infrastructure and Private Sector Productivity, in: OECD Economic Studies 17 (1991), 63-89; Kitterer, W./Schlag, C.-H.: Sind öffentliche Investitionen produktiv?, in: Finanzarchiv 52/4 (1995), 460-477; Thöne, M.: Wachstums- und nachhaltigkeitswirksame öffentliche Ausgaben (WNA), in: BMF, Monatsbericht März 2004, 73-79, hier 74 ff.

54 Für die Berechnung der Produktivität werden allein drei völlig verschiedene Methoden angewendet s. Mizutani, F./Tanaka, T.: Productivity Effects and Determinants of Public Infrastructure Investment, in: The Annals of Regional Science 2008 - online version, 1-30, hier 4 f.; zur Problematik und Methodik der Kosten-Nutzen Analysen im öffentlichen Bereich als einer der drei Methoden vgl. hier nur einführend Blankart, C. B., Finanzen, a. a. O, $419 \mathrm{ff}$.

55 Wiss. Beirat beim BMF: Gutachten zum Begriff der öffentlichen Investitionen, 1980, 48; Sachverständigenrat, a.a. O., 50.

56 Vgl. bereits Heun, W.: Art. 115, a.a.O, Rn. 22; dazu traten praktische Schwierigkeiten s. ebd. m.w. N. 
das crowding out. ${ }^{57}$ Die Zukunftsbelastung entsteht somit nicht, was regelmäßig in diesem Zusammenhang nicht thematisiert wird. Zweitens kann die Kreditaufnahme als Mittel antizyklischer Konjunktursteuerung sinnvoll sein. Es kann an dieser Stelle nicht die gesamte Problematik antizyklischer Finanzpolitik umfassend entfaltet werden, zumal es sich um ein hochumstrittenes und vermintes Feld handelt. Immerhin wird man aber abbreviaturhaft Folgendes festhalten können: Zum einen teilt heute niemand mehr die euphorischen Vorstellungen einer konjunkturellen Feinsteuerung durch den Staat, die Ende der 1960er Jahre in der Politik vorherrschten. Allein die Erfahrungen des Staatsversagens bei der Nachfragesteuerung sprechen dagegen. ${ }^{58}$ Diagnose und Prognosefehler sowie verfehlte Wirkungsabschätzungen, Handlungs- und Wirkungsverzögerungen mit der Folge zeitlicher Fehlsteuerung, begrenzte Wirkungsmöglichkeiten angesichts außenwirtschaftlicher Verflechtungen, Gewöhnungseffekte und Antizipation staatlicher Nachfragesteuerung reduzieren die Handlungs- und Steuerungsmöglichkeiten des Staates. Andererseits eliminiert die Möglichkeit des Staatsversagens noch nicht das Marktversagen, wie die derzeitige Krise drastisch vor Augen geführt hat. Allein die Wirkungen automatischer Stabilisatoren wie der Sozialversicherung reichen in schwerwiegenden Krisen nicht aus. Staatliche Ausgaben sind immer noch mit Multiplikatoreffekten verbunden, auch wenn die Wirkungen offenbar seit etwa 1980 zurückgegangen sind. ${ }^{59}$ Der Wirkungsgrad ist freilich auch und gerade in der derzeitigen Krise umstritten und hochgradig abhängig von dem jeweiligen ökonomischen Standpunkt. ${ }^{60}$ Schließlich bestreitet

57 Ebd., Rn. 24 m.w. N.

58 Grundlegende theoretische Einsichten betrafen v.a. die geldpolitische Steuerung und die Erkenntnis, dass das Faktum gleichbleibender oder erhöhter Arbeitslosigkeit bei ansteigender Inflationsrate durch die Einfügung der erwarteten Inflationsrate als Lageparameter in das Modell der Phillips-Kurve zu erklären ist, vgl. Friedman, M.: The Role of Monetary Policy, in: American Economic Review, 58/1 (1968), 1-17, hier 7 ff.; Phelps, E. S.: Money-Wage Dynamics and Labor Market Equilibrium, in: Journal of Political Economy, 76/4 (1968), 678-711; vgl. auch Welfens, P.J.J.: Theorie und Praxis angebotsorientierter Stabilitätspolitik, Baden-Baden, 1985, 70 ff., 24 ff.; s. ferner Starbatty, J.: Erfolgskontrolle der Globalsteuerung, Frankfurt/M., 1976, 93 ff.; Heun, W.: Staatshaushalt und Staatsleitung, Baden-Baden, 1989, $129 \mathrm{ff}$.

59 Vgl. Perotti, R.: Estimating the Effects of Fiscal Policy in OECD Countries, CEPR Working Paper 276, 2004.

60 Vgl. die Übersicht Hemming, R./Kell, M./Mahfouz, S.: The Effectiveness of Fiscal Policy in Stimulating Economic Activity - A Review of the Literature, IMF Working Paper WP/02/208, Dec. 2002; allgemein jetzt Sachverständigenrat: Die Zukunft nichts aufs Spiel setzen, Jahresgutachten 2009/10, $169 \mathrm{ff}$. Der Gegensatz zwischen neokeynesianischen und rationalen Erwartungen - Ansätzen wird geradezu idealtypisch widergespiegelt in den gegensätzlichen Untersuchungen von Romer, C./Bernstein, J.: The Job Impact of the American Recovery and Reinvestment Plan, 10.01. 2009 einerseits und Cogan, J.F. u. a.: New Keynesian versus Old Keynesian Government Spending Mutipliers, Feb. 2009. 
niemand ernsthaft, dass allein die durch die konjunkturell bedingte Verminderung der Einnahmen und Erhöhung der Sozialausgaben nicht prozyklisch verstärkt werden darf und somit ein deutlich erhöhter Kreditbedarf in einer Krise besteht. Angesichts dieser Umstände ist jedenfalls prinzipiell gegen die Regel des alten Art. 115 Abs. 1 GG wenig einzuwenden.

Vorwerfen ließ sich der alten Regelung allenfalls, dass die Formulierung „Störung des gesamtwirtschaftlichen Gleichgewichts“ zu offen war, um scharfe Begrenzungswirkungen zu entfalten. Der pauschale Vorwurf, die bisherige Regelung habe versagt ${ }^{61}$, ist aber völlig überzogen. Der Schuldenstand vor der jüngsten Finanzkrise ist wesentlich auf zwei Verschuldungsschübe zurückzuführen, nämlich im Gefolge der Rezession von 1975 und infolge der deutschen Wiedervereinigung. ${ }^{62}$ Beide Schübe wären auch in keiner Weise durch die neue rigidere Schuldenbremse verhindert worden. Im Übrigen ist die Verschuldungspolitik der Bundesrepublik gerade nicht als überzogen und überschäumend zu qualifizieren. Ohne die Wiedervereinigung läge der Schuldenstand deutlich unter dem Durchschnitt der OECD-Länder. Auch finden sich genügend Beispiele markanter Konsolidierung der Haushalte in westlichen Demokratien ohne rigide Verfassungsregeln. ${ }^{63}$

\section{Balanced Budget Amendments und Schuldenbremsen}

Die angeblichen Schwächen der relativ flexiblen Regelung des alten Art. 115 GG lassen den Blick auf striktere Begrenzungsregeln fallen, für die es vor allem in den USA und der Schweiz Vorbilder gibt, welche jetzt ihrerseits die Einführung einer strikten Schuldenbremse in Deutschland angeregt haben. Eine besonders lange Tradition haben Begrenzungsregeln in fast allen Bundesstaaten der USA, die sich allerdings im Einzelnen unterscheiden. Beinahe die Hälfte der Verfassungen schreibt strikt einen ausgeglichenen Haushalt vor, einige Staaten lassen eine Übertragung des Defizits in das folgende Haushaltsjahr zu, während andere den Schuldenstand begrenzen. ${ }^{64}$ Entscheidend ist freilich, dass die Budgetaus-

61 So etwa Wieland, J.: Staatsverschuldung als Herausforderung für die Finanzverfassung, in: Juristenzeitung, 61/15-16 (2006), 751-756, hier 752f.

62 Überblick über die Entwicklung seit 1950 etwa in: BMF: Finanzbericht 2008, $208 \mathrm{ff}$; speziell zur Wiedervereinigung Weltring, S.: Staatsverschuldung als Finanzierungsinstrument des deutschen Vereinigungsprozesses, Frankfurt/M., 1997, 37 ff.

63 S. bereits oben.

64 Die Typologie lässt sich noch weiter differenzieren: Vgl. die Überblicke U.S. General Accounting Office (GAO): Budget Issues, State Balanced Budget Practices, Dec. 1985; GAO: Balanced Budget Require- 
gleichsregeln fast immer lediglich für das operating budget und nur für die garantierte Schuld, also nicht für Haushalte aus zweckgebundenen Abgaben gelten. Der Vermögenshaushalt bleibt völlig ausgenommen. Deswegen wirken die Schuldenbegrenzungsregeln lediglich für ihren Geltungsbereich, wobei verfassungsrechtliche Verankerung und verfassungsgerichtliche Durchsetzungsmöglichkeit zusätzlich verstärkend wirken. Der Bereich der Investitionen ist mit dem Vermögenshaushalt aber ohnehin durch Kredite finanzierbar und außerdem kann ohne Schwierigkeiten in Schattenhaushalte ausgewichen werden. ${ }^{65}$ Bezogen auf die Verschuldung aller einzelstaatlichen Haushalte kommen empirische Untersuchungen daher durchgehend zu dem Ergebnis, dass die Begrenzungsregeln im Vergleich zu Einzelstaaten ohne derartige Regeln keine ersichtlich gewichtigen Effekte erzielen. ${ }^{66}$ Auf der Bundesebene sind - auch deswegen und wegen der ganz anderen Aufgaben - Versuche zur Einführung eines verfassungsrechtlichen Balanced Budget Amendment zu Recht gescheitert. ${ }^{67}$

Die jüngste deutsche Verfassungsänderung hat sich deshalb vielmehr an den gleichfalls neueren Regeln der Schweiz orientiert, die auch der Sachverständigenrat zur Übernahme empfohlen hatte. ${ }^{68}$ In der Schweiz kennen einige Kantonsverfassungen solche Regeln, ${ }^{69}$ seit 2002 aber ebenfalls die Schweizer Bundesverfassung in Art. 126, der 2001 in einer Volksabstimmung mit überwäl-

ments. State Experiences and Implications for the Federal Government, March 1993; Advisory Commission on Intergovernmental Relations (ACIR): Fiscal Discipline in the Federal System. National Reform and the Experience of the States, Washington, DC, 1987, $37 \mathrm{ff}$.

65 Deutlich herausgearbeitet bei Fisher, L.: The Effects of a Balanced Budget Amendment on Political Institutions, in: Journal of Law and Politics, 9/1 (1992), 89-104, hier $90 \mathrm{ff}$.; vgl. Saturno, J. V./Forgette, R. G.: The Balanced Budget Amendment: How Would It Be Enforced?, in: Public Budgeting \& Finance, 18/1 (1998), 33-53.

$66 \mathrm{Vgl}$. mit etwas unterschiedlicher Nuancierung Hagen, J.v.: A Note on the Empirical Effectiveness of Formal Fiscal Restraints, in: Journal of Public Economics, 44/2 (1991), 199-210; Bohn H./Inman, R.: Balanced Budget Rules and Public Deficits: Evidence from the U. S. States, NBER Working Paper 5533, 1996; Poterba, J.: State Responses to Fiscal Crises, The Effects of Budgetary Institutions and Politics, in: Journal of Political Economy, 102/4 (1994), 799-821.

67 Senate Joint Resolution 106, 104 Cong., 1 sess. 1995; zur Debatte vgl. U.S. Congress, House Committee on the Budget: The Balanced Budget Amendment, Hearings 2 vols 102 Cong., 2 sess. 1992; s. a. Saturno, J.V.: A Balanced Budget Constitutional Amendment: Background and Congressional Options, CRS-Report 97-379 GOV, 20.03. 1997.

68 Sachverständigenrat: Staatsverschuldung, a. a. O., $82 \mathrm{ff}$.

69 Vgl. dazu Kirchgässer, G.: Die Wirksamkeit von Schuldenbremsen, in: Schaltegger, C./Schaltegger, S. (Hg.): Perspektive der Schweizer Wirtschaftspolitik, Zürich, 2004, 107-123; Masoni, M.: Ausgabenbremse: der neue Weg des Kantons Tessin, in: ebd., 125-135. 
tigender Mehrheit angenommen worden ist. ${ }^{70}$ Ein Ausführungsgesetz setzt die Vorgaben um. ${ }^{71}$ Ein genauer Blick auf die verfassungsrechtliche Regelung offenbart allerdings erhebliche Spielräume für den Gesetzgeber. Der Bund ist lediglich pauschal verpflichtet „Ausgaben und Einnahmen auf Dauer im Gleichgewicht“ zu halten (Art. 126 Abs. 1). Die Ausgaben richten sich ,unter Berücksichtigung der Wirtschaftslage nach den geschätzten Einnahmen“ (Art. 126 Abs. 2). Zusätzlich kann der Betrag „,bei außerordentlichem Zahlungsbedarf angemessen erhöht werden“ (Art. 126 Abs. 3), „Mehrausgaben sind nur in den Folgejahren zu kompensieren“ (Art. 126 Abs. 4), was technisch über Ausgleichskonten erreicht wird ${ }^{72}$, während die Wirtschaftslage durch einen Konjunkturfaktor berücksichtigt wird ${ }^{73}$. Wie flexibel die Lösung sein kann, erwies sich gleich zu Beginn, als wegen einer Rezession die Regelung erst einmal für drei Jahre ausgesetzt wurde. ${ }^{74}$ Gleichwohl wirkt die Regelung prozyklisch ${ }^{75}$ und gilt zudem für ein Land von der Größe eines mittleren deutschen Bundeslandes ${ }^{76}$.

Nach vielfacher Kritik ${ }^{77}$ und der - verfehlten - Aufforderung des Bundesverfassungsgerichts zu einer ,grundlegenden Revision““78 hat im Rahmen der Föderalismus-Reform II der verfassungsändernde Gesetzgeber mit Geltung ab dem 1. August 2009 ebenfalls eine Schuldenbremse in das Grundgesetz (Art. 109 Abs. 3, 109a, 115 GG) inkorporiert. ${ }^{79}$ Danach sind einheitlich die Haushalte von Bund

70 Eidgenössische Finanzverwaltung/Colombier, C.: Eine Neubewertung der Schuldenbremse, Working Paper, 2. rev. version, Bern, 2004, 11; Bodmer, F.: The Swiss Debt Brake: How it Works and What Can Go Wrong, in: Schweizerische Zeitschrift für Volkswirtschaft und Statistik, 142/3 (2006), 307-330, hier 307.

71 Art. 13 ff. (3. Kap., 2. Abschn.) des (jeweiligen) Bundesgesetzes über den eidgenössischen Finanzhaushalt.

72 Art. 16, 17 Finanzhaushaltsgesetz.

73 Art. 13 Finanzhaushaltsgesetz; zur Problematik des zur Glättung des Entwicklungstrends verwendeten Filters s. Colombier, C., a. a. O., $16 \mathrm{ff} ., 24 \mathrm{ff}$.

74 Bodmer, F., a. a. O., 307.

75 Vgl. Sachverständigenrat: Staatsverschuldung, a.a. O., $157 \mathrm{ff}$.

76 Ende 2008 belief sich die Zahl der ständigen Wohnbevölkerung auf 7,7 Mio. Personen; Eidgenössisches Bundesamt für Statistik, Medienmitteilung v. 26.02. 2009; eine ganze Reihe von Kantonen hat weniger als 50.000 Einwohner.

77 Vgl. hier nur jüngst z. B. Tappe, H.: Die neue Schuldenbremse im Grundgesetz, in: Die öffentliche Verwaltung, 62/21 (2009), 881-890, hier $882 \mathrm{ff}$.

78 BVerfGE 119, 96 ff., hier $141 \mathrm{ff}$;; s. ferner neben Sachverständigenrat: Staatsverschuldung, a.a.O., 73 ff.; Wissenschaftlicher Beirat beim Bundesministerium für Wirtschaft und Technologie: Zur Begrenzung der Staatsverschuldung nach Art. 115 GG und zur Aufgabe des Stabilitäts- und Wachstumsgesetzes, 2008, $19 \mathrm{ff}$.

79 Ges. v. 29.07. 2009 (BGB1. I, 2248); Entwurf: BT-Drs. 16/12410. 
und Ländern grundsätzlich ohne Einnahmen aus Krediten auszugleichen. Allein dem Bund wird eine reguläre Kreditaufnahme in Höhe von 0,35\% des BIP eine völlig willkürlich gegriffene Zahl - gestattet. Für die Länder gilt das Verbot der strukturellen Verschuldung aber uneingeschränkt. Ausnahmen für Bund und Länder sollen allerdings noch zulässig sein ,zur im Auf- und Abschwung symmetrischen Berücksichtigung der Auswirkungen einer von der Normallage abweichenden konjunkturellen Entwicklung" sowie im Falle von Naturkatastrophen und sonstigen unkontrollierbaren und finanziell erheblichen Notsituationen. Dabei soll aber - wieder nach Schweizer Vorbild - eine Tilgungsregelung vorgesehen werden. Der Bund soll ab 2013, die Länder ab 2020 strikt an das Verbot gebunden $\operatorname{sein}^{80}$

Die Regelung stößt weithin auf Zustimmung ${ }^{81}$, kritisch werden am ehesten die Wirksamkeit in Zweifel gezogen ${ }^{82}$, die sprachliche Form gerügt ${ }^{83}$ oder Details problematisiert. Am deutlichsten wird noch die Inpflichtnahme der Bundesländer durch den Bund als unzulässiger Eingriff in die Staatlichkeit der Länder nach Art. 79 Abs. 3 GG angegriffen. ${ }^{84}$ Das ist angesichts der bestehenden und nie angezweifelten Vorgaben des Art. 109 Abs. 2 GG und des darauf beruhenden Haushaltsgrundsätzegesetzes sowie der Garantenpflichten des Bundes wenig einsichtig, zumal die unbegrenzte Verschuldungsfähigkeit wohl kaum zum irrevisiblen Kernbestand der Finanzautonomie der Länder zu rechnen ist. Demgegenüber sind ökonomische und rechtliche Rechtfertigung sowie die Wirksamkeit dieser Schuldenbremse in höchstem Maße fragwürdig. Das Ziel der Nachhaltigkeit erfordert kein prinzipielles Verbot, sondern lediglich eine relative Beschränkung bezogen auf das BIP. Da auch die Koppelung der Kredite an die Investitionen aufgehoben worden ist, entfällt jeder verfassungsrechtliche Anreiz zu staat-

80 Die komplizierten Übergangsregeln enthält Art. 143d GG.

81 Exemplarisch: Seiler, C.: Konsolidierung der Staatsfinanzen mithilfe der neuen Schuldenregel, in: Juristenzeitung, 64/14 (2009), 721-728; Pünder, H.: Gerechte Lastenverteilung zwischen den Generationen, in: Deutsches Verwaltungsblatt, 123/15 (2008), 946-956.

82 Korioth, S.: Das neue Staatsschuldenrecht - zur zweiten Stufe der Föderalismusreform, in: Juristenzeitung, 64/14 (2009), 729-737, hier 736.

83 Klein, H.H.: Ein erbärmliches Zeugnis, in: Frankfurter Allgemeine Zeitung v. 07.05. 2009; so bereits der Bundestagspräsident Lammert in: Frankfurter Allgemeine Zeitung v. 23.04. 2009; s. a. Tappe, H., a.a.O, 889; Selmer, P.: Die Föderalismusreform II - Ein verfassungsrechtliches monstrum simile, in: Neue Zeitschrift für Verwaltungsrecht, 28/20 (2009), 1255-1262, hier 1259 ff.; Korioth, S., a.a.O., $736 \mathrm{f}$.

84 Etwa Fassbender, B.: Eigenstaatlichkeit und Verschuldungsfähigkeit der Länder, in: Neue Zeitschrift für Verwaltungsrecht, 28/12 (2009), 737-741; Hancke, J.: Defizitbegrenzung im Bundesstaat, in: Deutsches Verwaltungsblatt, 124/10 (2009), 621-627, hier 626. 
lichen Investitionen ${ }^{85}$, zumal dieser Budgetposten am wenigsten rechtlich gebunden und damit für den Haushaltsgesetzgeber disponibel ist. Vermutlich wird die staatliche Investitionsquote deshalb deutlich zurückgehen, da das Verfassungsrecht falsche Anreize setzt. Außerdem entfällt jedenfalls im Inland langfristig die stabilste Anlagemöglichkeit, was die Volatilität der Finanzmärkte nicht verringert und deutsche Anleger zum Ausweichen auf ausländische Staatsanleihen zwingt. ${ }^{86}$ Es besteht ferner die Gefahr einer prozyklischen Budgetpolitik, da die Spielräume zu rigide begrenzt werden. Das ist auch im Rahmen der europäischen Rahmenbedingungen einer einheitlichen Geldpolitik, die sich vorrangig am Inflationsziel orientiert, und der Währungseinheit im Euro-Raum ohne gemeinsame Finanz- und Wirtschaftspolitik eine zu scharfe Beschneidung fiskalpolitischer Handlungsfähigkeit. ${ }^{87}$

Es kommt hinzu, dass die Schuldenbremse vielleicht nicht völlig, aber doch weithin wirkungslos bleiben wird. Das beginnt mit den langen Übergangsfristen: Hier hat die gegenwärtige zu Lasten der zukünftigen Politikergeneration verfassungsrechtliche Barrieren errichtet. Zweitens gibt es kaum Sanktionsmöglichkeiten. Das Bundesverfassungsgericht ist zwar rechtlich, aber nicht ökonomisch sachverständig und deshalb weithin mit der Beurteilung überfordert, ein Urteil ergeht zudem zu spät und hat kaum praktische Auswirkungen. ${ }^{88}$ Schließlich eröffnet auch die gegenwärtige Regelung noch genügend Ausweichmöglichkeiten. Trotz der Erfassung der Abweichungen der tatsächlichen von der erlaubten Kreditaufnahme im Kontrollkonto sind haushaltstechnische Tricks nicht ausgeschlossen. Vor allem anderen jedoch bleibt ein Tor für die Umgehung des Verschuldungsverbots weit geöffnet: die Schattenhaushalte. Zwar wird das Kreditverbot auch auf neue Sondervermögen erstreckt, aber zum einen bleiben am 31 . Dezember 2010 bestehende Kreditermächtigungen für bereits eingerichtete Sondervermögen erhalten. ${ }^{89}$ Zum anderen werden weder bisher ${ }^{90}$ noch zukünftig juristisch selbständige Einrichtungen, auch wenn sie vom Bund finanziert werden und der Bund für deren Verbindlichkeiten haftet, überhaupt von der Schul-

85 Vgl. Bofinger, P.: Markt, a. a. O, $198 \mathrm{ff}$.

86 Auf diese Ausweichreaktion weist auch hin: ebd., 196.

87 Vgl. Sachverständigenrat: Staatsverschuldung, a. a. O., $157 \mathrm{ff}$.

88 Vgl. auch Tappe, H., a. a. O., 890.

89 Art. 143d Abs. 1 Satz 2 GG; s. a. Korioth, S., Staatschuldenrecht, a. a. O, 733.

90 Heun, W., Art. 115, a. a. O., Rn. 36; Siekmann, H., in: Sachs, M. (Hg.): GG-Kommentar, 5. Aufl., München, 2009, Art. 115, Rn. 60. 
denbremse erfasst. ${ }^{91}$ Die selbständigen Sozialversicherungsträger könnten daher unbeschadet der Schuldenbremse Kredite aufnehmen - die der Bund dann bedient. Die Erfahrungen der US-Bundesstaaten sprechen für diesen Ausweg - und die neue Koalition hat, bevor die Schuldenbremse überhaupt greift, schon auf diesen Weg in Milliardenhöhe zur Finanzierung von unnötigen Steuersenkungen $^{92}$ zurückgreifen wollen und ist nur wegen des politischen Drucks davor zurückgeschreckt ${ }^{93}$. Welche Barriere die Schuldenbremse im Ernstfall entfalten wird, kann sich danach jeder unschwer ausmalen.

\section{Resümee}

Drei Ziele müssen materielle verfassungsrechtliche Verschuldungsregeln unter optimalen Voraussetzungen verfolgen und erfüllen. Erstens sollte langfristig die fiskalische Nachhaltigkeit gesichert werden. Zweitens sollte die intertemporale Lastenverschiebung infolge des crowding out durch staatliche Investitionen kompensiert werden. Drittens muss das Instrument der Kreditaufnahme im Rahmen einer antizyklischen Haushaltspolitik im Grundsatz erhalten bleiben, ohne zu dem Versuch einer Feinsteuerung anzuspornen. Die bisherige Kombination der Begrenzung des Defizits auf die Höhe der Investitionsausgaben durch Art. $115 \mathrm{GG}$ und auf $3 \%$ durch das Europarecht sowie die Ausnahmeregelung bei einer Störung des gesamtwirtschaftlichen Gleichgewichts in Deutschland haben dieses Anforderungsprofil im Grunde recht gut ausgefüllt - trotz einiger Mängel im Detail. Die neue Schuldenbremse verfehlt das Profil dagegen deutlich. Sie schießt teilweise über das Ziel hinaus und ist zu rigide, eine Kompensation wird gar nicht angestrebt. Sie provoziert daher Fehlsteuerung und Ausweichmanöver außerhalb ihres unmittelbaren Geltungsbereichs. Die jüngste, auch aus Populismus geborene deutsche Verfassungsreform ist deshalb in seltenem Maß formal und inhaltlich missglückt und irregeleitet.

91 Auch gesehen von Tappe, H., a. a. O., 889.

92 Vgl. Sachverständigenrat: Zukunft, a.a.O., 185 ff.; Deutsche Bundesbank: Monatsbericht Nov. 2009, 70.

93 Vgl. FAZ.net vom 20.10. 2009 und 21.10. 2009; wegen der Übergangsregelung des Art. 143d GG sollte sogar noch ein Sondervermögen geschaffen werden, das mit Krediten in Höhe von 60 Mio. $€$ Zuschüsse zu den Sozialleistungen finanzieren sollte. 